\title{
RECURSIVE FORMULAS RELATED TO THE SUMMATION OF THE MÖBIUS FUNCTION
}

\author{
MANUEL BENITO AND JUAN L. VARONA
}

\begin{abstract}
For positive integers $n$, let $\mu(n)$ be the Möbius function, and $M(n)$ its sum $M(n)=\sum_{k=1}^{n} \mu(k)$. We find some identities and recursive formulas for computing $M(n)$; in particular, we present a two-parametric family of recursive formulas.
\end{abstract}

\section{INTRODUCTION}

The well-known Möbius function $\mu(n)$ is defined, for positive integers $n$, as

$$
\mu(n):= \begin{cases}1 & \text { if } n=1, \\ (-1)^{k} & \text { if } n \text { is a product of } k \text { different prime numbers } \\ 0 & \text { if there exists a prime } p \text { such that } p^{2} \text { divides } n\end{cases}
$$

(see [1, Chapter 2]). Then, for every real number $x \geq 0$, the summation of the Möbius function is defined by taking

$$
M(x)=M(\lfloor x\rfloor):=\sum_{k=1}^{\lfloor x\rfloor} \mu(k) .
$$

In what follows, and as usually, we refer to $M(x)$ as the Mertens function, although, before F. Mertens (who used it in 1897, see [2]), T. J. Stieltjes already had introduced this function in his attempts to prove the Riemann Hypothesis (see [3, Lettre 79, p. 160-164], dated in 1885).

The behaviour of $M(x)$ is rather erratic and difficult of analyze, but it is very important in analytic number theory. In 1912, J. E. Littlewood [4] proved that the Riemann Hypothesis is equivalent to this fact:

$$
|M(x)|=O\left(x^{1 / 2+\varepsilon}\right), \quad \text { when } x \rightarrow \infty, \quad \text { for every } \varepsilon>0 ;
$$

in relation to this subject, see also [5]. Of course, it is not yet known if (1) is true or false. Previously, in 1897, Mertens [2] had given a table of values of $M(n)$ for $1 \leq n \leq 10000$. Relying on this table, he conjectured that, for $x>1$,

$$
|M(x)|<\sqrt{x} \text {. }
$$

This conjecture was disproved, in 1985, by A. M. Odlyzko and H. te Riele [6], but they did not find an explicit counterexample. Actually, for every value of $M(n)$ computed up to that date, always happened $|M(n)|<0.6 \sqrt{n}$. In 1987, J. Pintz [7] proved that the Mertens conjecture is false for some $n<\exp \left(3.21 \times 10^{64}\right)$; and this was improved further recently in 2006 by T. Kotnik and H. te Riele [8], who showed that the Mertens conjecture is false for some $n<\exp \left(1.59 \times 10^{40}\right)$. More studies about the order of the Mertens function can

2000 Mathematics Subject Classification. Primary 11A25.

Key words and phrases. Möbius function, summation of the Möbius function, Mertens function.

Research of the second author supported by grant MTM2006-13000-C03-03 of the DGI. 
be found in [9] and [10]. Nowadays, to find an explicit counterexample of the Mertens conjecture is yet a very pursued result in number theory, and it generally believed that no counterexample will be found for $n<10^{20}$.

To evaluate $M(n)$, a big quantity of recursive formulas appear in the mathematical literature. For instance, Stieltjes [3, Letter 79, p. 163] proved the expression

$$
\sum_{k \leq \sqrt{n}}(-1)^{k-1} M(n / k)=-1+M(\sqrt{n}) z(\sqrt{n})-\sum_{k \leq \sqrt{n}} z(n / k) \mu(k)
$$

where $z(x)=0$ if $\lfloor x\rfloor$ is even and 1 if it is odd; some other recursive formulas appear in the famous Primzahlen of E. Landau [11]. In 1996, M. Deléglise and J. Rivat [12], used an algorithm derived from the recurrence formula

$$
M(x)=M(u)-\sum_{a \leq u} \mu(a) \sum_{\frac{u}{a}<b \leq \frac{x}{a}} M\left(\frac{x}{a b}\right)
$$

(being $1 \leq u \leq x)$ to evaluate $M\left(10^{16}\right)=-3195437$. More recursive formulas can be found in [13], and [14]; also, a large number of further references to related studies, including a nice historical review, are given in [15].

The aim of this paper is to prove different identities and recursive formulas satisfied by the Mertens function $M$. We devote to this end sections 2, 3 and 4; see Theorems 2, 3,6, 9 , and 10. For instance, in Theorem 3 we present a formula to evaluate $M(n)$ similar to the one given by its definition, but with only $\left\lfloor\frac{n}{3}\right\rfloor$ summands. Also, let us note the interesting expansion for $2 M(n)+3$ that appears in Theorem 6 , as well as the properties of the involved coefficients, studied below; they will lead us to Theorems 9 and 10. In particular, Theorem 10 gives a two-parametric family of recursive formulas for computing the Mertens function. As long as we know, all the "theorems" that we present in these sections are new; however, some of the "propositions" are already known, and we have included them by completeness.

Finally, in section 5 , we study some properties of a function (that we will denote $H(n, m)$ ) related with the ones that appear in the previous sections; in particular, we prove the periodicity of this function.

\section{Formulas IN Which ONLY $M$ APPEARS}

Let us begin by recalling the following well-known property of the Möbius function:

$$
\sum_{d \mid n} \mu(d)=\left\{\begin{array}{lll}
1 & \text { if } & n=1 \\
0 & \text { if } & n>1
\end{array}\right.
$$

Indeed, it is trivial for $n=1$. And, for $n>1$, if $n=\prod_{j=1}^{k} p_{j}^{\alpha_{j}}>1\left(p_{j}\right.$ primes, $p_{j} \neq p_{i}$ for $j \neq i$ ), then

$$
\sum_{d \mid n} \mu(d)=\left(\begin{array}{l}
k \\
0
\end{array}\right)-\left(\begin{array}{l}
k \\
1
\end{array}\right)+\cdots+(-1)^{k}\left(\begin{array}{l}
k \\
k
\end{array}\right)=(1-1)^{k}=0 .
$$

The identity (4) allows to find a way of relating the value $M(n)$ with the values of $M(m)$, with $m$ less than $n$. This result, also known (and whose proof we reproduce by completeness), is the following:

Proposition 1. For every positive n, the Mertens function verifies

$$
1=\sum_{a=1}^{n} M\left(\frac{n}{a}\right)
$$


Proof. Actually, we will prove (5) also for real numbers $x \geq 1$. From the definition $M(x)=$ $\sum_{k \leq x} \mu(k)$, we have

$$
\sum_{a=1}^{\lfloor x\rfloor} M\left(\frac{x}{a}\right)=\sum_{a=1}^{\lfloor x\rfloor} \sum_{b=1}^{\left\lfloor\frac{x}{a}\right\rfloor} \mu(b) .
$$

If $a b=k$, then $a \mid k$ and, moreover, when the values of $a$ and $b$ vary, $k$ takes the values $1,2, \ldots,\lfloor x\rfloor$. Then, we have

$$
\sum_{a=1}^{\lfloor x\rfloor} \sum_{b=1}^{\left\lfloor\frac{x}{a}\right\rfloor} \mu(b)=\sum_{1 \leq k \leq\lfloor x\rfloor} \sum_{a \mid k} \mu(a) .
$$

By applying (4), we get (5).

Of course, from (5) we obtain the following recursive formula satisfied by $M(n)$ :

$$
M(n)=1-\sum_{a=2}^{n} M\left(\frac{n}{a}\right)
$$

which is essentially one of the recursive formulae used by Neubauer [13] to compute $M(n)$ up to $10^{10}$. Moreover, let us note that (4) and (5) were used by Deléglise and Rivat [12] to find the identity (3).

In (6), $n$ summands appear. In the following theorem, we reduce the number of summands up to $\left\lfloor\frac{n-1}{2}\right\rfloor$.

Theorem 2. If $n \geq 3$, then

$$
M(n)=-\sum_{a=1}^{\left\lfloor\frac{n-1}{2}\right\rfloor} M\left(\frac{n}{2 a+1}\right) .
$$

Proof. If $n=2 m$ with $m>1$, by applying (6) and (5), we get

$$
\begin{aligned}
M(2 m) & =1-\sum_{a=2}^{2 m} M\left(\frac{2 m}{a}\right)=\sum_{a=1}^{m} M\left(\frac{m}{a}\right)-\sum_{a=2}^{2 m} M\left(\frac{2 m}{a}\right) \\
& =-\sum_{a=1}^{m-1} M\left(\frac{2 m}{2 a+1}\right)=-\sum_{a=1}^{\left\lfloor\frac{n-1}{2}\right\rfloor} M\left(\frac{n}{2 a+1}\right) .
\end{aligned}
$$

For the case $n=2 m+1$, let us first note that the greatest remainder that can be obtained when $m$ is divided by $a$ is $a-1$, and, moreover

Thus, it is clear that

$$
\frac{a-1}{a}+\frac{1}{2 a}=\frac{2 a-2+1}{2 a}=\frac{2 a-1}{2 a}<1 .
$$

$$
M\left(\frac{2 m+1}{2 a}\right)=M\left(\frac{m}{a}+\frac{1}{2 a}\right)=M\left(\frac{m}{a}\right) .
$$

Then, by applying (6), (5), and this fact, we get

$$
\begin{aligned}
M(2 m+1) & =1-\sum_{a=2}^{2 m+1} M\left(\frac{2 m+1}{a}\right)=\sum_{a=1}^{m} M\left(\frac{m}{a}\right)-\sum_{a=2}^{2 m+1} M\left(\frac{2 m+1}{a}\right) \\
& =-\sum_{a=1}^{m} M\left(\frac{2 m+1}{2 a+1}\right)=-\sum_{a=1}^{\left\lfloor\frac{n-1}{2}\right\rfloor} M\left(\frac{n}{2 a+1}\right) .
\end{aligned}
$$




\section{Formulas IN WHICH ONLY $\mu$ APPEARS}

In the following theorem, we expand $M(n)$ as a sum with $\left\lfloor\frac{n}{3}\right\rfloor$ summands, in which only $\mu$ and the integer-part function appear. In particular, this result provides a more efficient way to compute $M(n)$ than just to use its definition $M(n)=\sum_{k=1}^{n} \mu(k)$.

Theorem 3. If $n \geq 3$, then

$$
M(n)=-\sum_{k=1}^{\left\lfloor\frac{n}{3}\right\rfloor}\left\lfloor\frac{n-k}{2 k}\right\rfloor \mu(k) .
$$

Proof. Let us remind (7) in Theorem 2. The greatest value achieved by $\left\lfloor\frac{n}{2 a+1}\right\rfloor$ is $\left\lfloor\frac{n}{3}\right\rfloor$. Moreover, $\left\lfloor\frac{n}{2 a+1}\right\rfloor$ takes value $k$ if

$$
k \leq \frac{n}{2 a+1}<k+1
$$

i.e.,

$$
\frac{n-(k+1)}{2(k+1)}<a \leq \frac{n-k}{2 k}
$$

In this way, $\left\lfloor\frac{n}{2 a+1}\right\rfloor=k$ for $\left\lfloor\frac{n-k}{2 k}\right\rfloor-\left\lfloor\frac{n-(k+1)}{2(k+1)}\right\rfloor$ values of $a$.

As a consequence,

$$
\begin{aligned}
& M(n)=-\sum_{k=1}^{\left\lfloor\frac{n}{3}\right\rfloor}\left(\left\lfloor\frac{n-k}{2 k}\right\rfloor-\left\lfloor\frac{n-(k+1)}{2(k+1)}\right\rfloor\right) M(k) \\
& =-\left(\left(\left\lfloor\frac{n-1}{2}\right\rfloor-\left\lfloor\frac{n-2}{2 \cdot 2}\right\rfloor\right) M(1)+\left(\left\lfloor\frac{n-2}{2 \cdot 2}\right\rfloor-\left\lfloor\frac{n-3}{2 \cdot 3}\right\rfloor\right) M(2)+\cdots\right. \\
& \left.+\left(\left\lfloor\frac{n-\left\lfloor\frac{n}{3}\right\rfloor}{2\left\lfloor\frac{n}{3}\right\rfloor}\right\rfloor-\left\lfloor\frac{n-\left\lfloor\frac{n}{3}\right\rfloor-1}{2\left(\left\lfloor\frac{n}{3}\right\rfloor+1\right)}\right\rfloor\right) M\left(\left\lfloor\frac{n}{3}\right\rfloor\right)\right) \\
& =-\left(\left\lfloor\frac{n-1}{2}\right\rfloor \mu(1)+\left\lfloor\frac{n-2}{2 \cdot 2}\right\rfloor \mu(2)+\left\lfloor\frac{n-3}{2 \cdot 3}\right\rfloor \mu(3)+\cdots\right. \\
& \left.+\left\lfloor\frac{n-\left\lfloor\frac{n}{3}\right\rfloor}{2\left\lfloor\frac{n}{3}\right\rfloor}\right\rfloor \mu\left(\left\lfloor\frac{n}{3}\right\rfloor\right)-\left\lfloor\frac{n-\left\lfloor\frac{n}{3}\right\rfloor-1}{2\left(\left\lfloor\frac{n}{3}\right\rfloor+1\right)}\right\rfloor M\left(\left\lfloor\frac{n}{3}\right\rfloor\right)\right) .
\end{aligned}
$$

Now, let us observe

$$
\frac{n-\left\lfloor\frac{n}{3}\right\rfloor-1}{2\left(\left\lfloor\frac{n}{3}\right\rfloor+1\right)}=\left\{\begin{array}{lll}
\frac{3 m-m-1}{2 m+2}=\frac{2 m-1}{2 m+2}<1 & \text { if } & n=3 m, \\
\frac{3 m+1-m-1}{2 m+2}=\frac{2 m}{2 m+2}<1 & \text { if } & n=3 m+1, \\
\frac{3 m+2-m-1}{2 m+2}=\frac{2 m+1}{2 m+2}<1 & \text { if } & n=3 m+2,
\end{array}\right.
$$

and so

$$
\left\lfloor\frac{n-\left\lfloor\frac{n}{3}\right\rfloor-1}{2\left(\left\lfloor\frac{n}{3}\right\rfloor+1\right)}\right\rfloor=0 .
$$

Then, (8) follows.

The following result relates the value of $\mu(n)$ to the values of $\mu(m)$ for $1 \leq m<n$. Actually, this result is already known (see [1, Theorem 3.12]), although the proof that we 
make in this paper is different and, perhaps, new; here, we use an argument similar to the one used in the proof of Theorem 3.

Proposition 4. The Möbius function satisfies

$$
1=\sum_{k=1}^{n}\left\lfloor\frac{n}{k}\right\rfloor \mu(k) .
$$

Proof. By Proposition 1, $1=\sum_{a=1}^{n} M\left(\frac{n}{a}\right)$. Here, we have $\left\lfloor\frac{n}{a}\right\rfloor=k$ if and only if

$$
k \leq \frac{n}{a}<k+1,
$$

i.e.,

$$
\frac{n}{k+1}<a \leq \frac{n}{k}
$$

and so $M\left(\frac{n}{a}\right)=M\left(\left\lfloor\frac{n}{a}\right\rfloor\right)=M(k)$ for $\left\lfloor\frac{n}{k}\right\rfloor-\left\lfloor\frac{n}{k+1}\right\rfloor$ values of $a$.

Then

$$
\begin{aligned}
1 & =\sum_{a=1}^{n} M\left(\frac{n}{a}\right)=\sum_{k=1}^{n}\left(\left\lfloor\frac{n}{k}\right\rfloor-\left\lfloor\frac{n}{k+1}\right\rfloor\right) M(k) \\
& =\left(\left\lfloor\frac{n}{1}\right\rfloor-\left\lfloor\frac{n}{2}\right\rfloor\right) M(1)+\left(\left\lfloor\frac{n}{2}\right\rfloor-\left\lfloor\frac{n}{3}\right\rfloor\right) M(2)+\cdots+\left(\left\lfloor\frac{n}{n}\right\rfloor-\left\lfloor\frac{n}{n+1}\right\rfloor\right) M(n) \\
& =\left\lfloor\frac{n}{1}\right\rfloor+\left\lfloor\frac{n}{2}\right\rfloor(M(2)-M(1))+\cdots+\left\lfloor\frac{n}{n}\right\rfloor(M(n)-M(n-1))-\left\lfloor\frac{n}{n+1}\right\rfloor M(n) \\
& =\left\lfloor\frac{n}{1}\right\rfloor \mu(1)+\left\lfloor\frac{n}{2}\right\rfloor \mu(2)+\cdots+\left\lfloor\frac{n}{n}\right\rfloor \mu(n)=\sum_{k=1}^{n}\left\lfloor\frac{n}{k}\right\rfloor \mu(k) .
\end{aligned}
$$

Now, let us prove another expansion of 1 as a sum of $\mu$ 's. We will use this result in the proof of Theorem 6 .

Proposition 5. For $n \geq 3$, the Möbius function satisfies

$$
1=\sum_{k=1}^{\left\lfloor\frac{n}{3}\right\rfloor}\left\lfloor\frac{n}{3 k}\right\rfloor \mu(k) .
$$

Proof. First, let us suppose $n=3 m$. By Proposition 4, we have

$$
1=\sum_{k=1}^{m}\left\lfloor\frac{m}{k}\right\rfloor \mu(k)=\sum_{k=1}^{\frac{n}{3}}\left\lfloor\frac{3 m}{3 k}\right\rfloor \mu(k)=\sum_{k=1}^{\left\lfloor\frac{n}{3}\right\rfloor}\left\lfloor\frac{n}{3 k}\right\rfloor \mu(k) .
$$

If $n=3 m+1$,

$$
\sum_{k=1}^{\left\lfloor\frac{n}{3}\right\rfloor}\left\lfloor\frac{n}{3 k}\right\rfloor \mu(k)=\sum_{k=1}^{m}\left\lfloor\frac{3 m+1}{3 k}\right\rfloor \mu(k)=\sum_{k=1}^{m}\left\lfloor\frac{m}{k}\right\rfloor \mu(k)=1,
$$

because, in

$$
\left\lfloor\frac{3 m+1}{3 k}\right\rfloor=\left\lfloor\frac{m}{k}+\frac{1}{3 k}\right\rfloor,
$$

the remainder when $m$ is divided by $k$ is always less or equal than $k-1$; and, by being

$$
\frac{k-1}{k}+\frac{1}{3 k}=\frac{3 k-2}{3 k}<1,
$$


we have

$$
\left\lfloor\frac{3 m+1}{3 k}\right\rfloor=\left\lfloor\frac{m}{k}\right\rfloor
$$

If $n=3 m+2$,

$$
\left\lfloor\frac{3 m+2}{3 k}\right\rfloor=\left\lfloor\frac{m}{k}+\frac{2}{3 k}\right\rfloor=\left\lfloor\frac{m}{k}\right\rfloor
$$

by being

$$
\frac{k-1}{k}+\frac{2}{3 k}=\frac{3 k-1}{3 k}<1
$$

thus,

$$
\sum_{k=1}^{\left\lfloor\frac{n}{3}\right\rfloor}\left\lfloor\frac{n}{3 k}\right\rfloor \mu(k)=\sum_{k=1}^{m}\left\lfloor\frac{3 m+2}{3 k}\right\rfloor \mu(k)=\sum_{k=1}^{m}\left\lfloor\frac{m}{k}\right\rfloor \mu(k)=1 .
$$

Then, we establish the following result, in which, as in Theorem 3, we show an expansion of $M(n)$ as a sum of $\left\lfloor\frac{n}{3}\right\rfloor$ summands; in every summand, only the Möbius function and a coefficient (related to the integer-part function) appear. This will be a fruitful result, because, later in this paper, we will find some alternative formulas and interesting properties for the coefficients.

Theorem 6. For $n \geq 3$, we have

$$
2 M(n)+3=\sum_{k=1}^{\left\lfloor\frac{n}{3}\right\rfloor} g(n, k) \mu(k)
$$

with

$$
g(n, k)=3\left\lfloor\frac{n}{3 k}\right\rfloor-2\left\lfloor\frac{n}{2 k}-\frac{1}{2}\right\rfloor .
$$

Proof. Let us add 2 times the expansion for $M(n)$ in Theorem 3 plus 3 times the expansion for 1 in Proposition 5.

The next result presents an alternative way for computing $g(n, k)$ :

Proposition 7. For $k>0$ and $n \geq 0$, let us take $n_{0}$ such that

$$
n \equiv n_{0} \quad \bmod 6 k, \quad 0 \leq n_{0}<6 k .
$$

Then

$$
g(n, k)=\left\{\begin{array}{lll}
2 & \text { if } & 0 \leq n_{0}<k \\
0 & \text { if } & k \leq n_{0}<3 k \\
1 & \text { if } & 3 k \leq n_{0}<5 k \\
-1 & \text { if } & 5 k \leq n_{0}<6 k
\end{array}\right.
$$

Proof. Le us decompose $n=n_{0}+6 k n_{1}$, with $0 \leq n_{0}<6 k$. By (11),

$$
g(n, k)=3\left\lfloor\frac{n_{0}+6 k n_{1}}{3 k}\right\rfloor-2\left\lfloor\frac{n_{0}+6 k n_{1}-k}{2 k}\right\rfloor .
$$

Then, it is clear that

$$
\begin{array}{ll}
\text { if } \quad 0 \leq n_{0}<k, & g(n, k)=6 n_{1}-2\left\lfloor\frac{6 k\left(n_{1}-1\right)}{2 k}+\frac{5 k+n_{0}}{2 k}\right\rfloor=2 ; \\
\text { if } \quad k \leq n_{0}<3 k, & g(n, k)=6 n_{1}-6 n_{1}=0 ; \\
\text { if } \quad 3 k \leq n_{0}<5 k, & g(n, k)=3+6 n_{1}-6 n_{1}-2=1 ; \\
\text { if } \quad 5 k \leq n_{0}<6 k, & g(n, k)=3+6 n_{1}-6 n_{1}-4=-1 .
\end{array}
$$




\section{Formulas in Which Both $M$ AND $\mu$ APPEAR}

Let us consider the function $g(n, k)$ for fixed $n$, i.e., as a function of $k$. In the following proposition, we show how $g(n, k)$ is constant when $k$ varies a certain interval.

Proposition 8. Let $a$ and $n$ be positive integers, with $a<n$. When $k$ varies in the interval

$$
\frac{n}{a+1}<k \leq \frac{n}{a}
$$

the value of $g(n, k)$ remains constant. This value depends only upon the remainder of a modulus 6 .

Proof. Let us decompose $a=a_{0}+6 a_{1}$ with $0 \leq a_{0}<6$. If $\frac{n}{a+1}<k \leq \frac{n}{a}$, then $k a \leq n<$ $k(a+1)$ and so

$$
k a_{0}+6 k a_{1} \leq n<k\left(a_{0}+6 a_{1}+1\right) .
$$

Thus, $n=n_{0}+6 k a_{1}$ for some $n_{0}$ verifying $0 \leq n_{0}<6 k$. By substituting this value of $n$ in (13), it becomes

$$
k a_{0} \leq n_{0}<k a_{0}+k .
$$

By (12), $g(n, k)$ takes the same value for all $n_{0}$ that satisfies (14), and this value of $g(n, k)$ depends only on $a_{0}$.

As a consequence of Proposition 8, we can define the function

$$
h(a)=g(n, k) \text { for } \quad \frac{n}{a+1}<k \leq \frac{n}{a} .
$$

By using (12) (pay attention to $a_{0}$ in the proof of Proposition 8), $h(a)$ takes these values:

$$
h(a)=\left\{\begin{array}{lll}
2, & \text { if } \quad a \equiv 0 \quad \bmod 6 \\
0, & \text { if } \quad a \equiv 1 \quad \text { mod } 6 \\
0, & \text { if } \quad a \equiv 2 \bmod 6 \\
1, & \text { if } \quad a \equiv 3 \bmod 6 \\
1, & \text { if } \quad a \equiv 4 \bmod 6 \\
-1, & \text { if } \quad a \equiv 5 \bmod 6
\end{array}\right.
$$

Now, we will split the sum in (10) in two parts, introducing a parameter $r$. The first part will consist in the $\left\lfloor\frac{n}{r+1}\right\rfloor$ first terms of the sum in (10). The second part will be, of course, the summands that remain; they will be manipulated in such way that we will get $r$ sumands in which only the functions $h$ and $M$ appear. We can say that this is a mixed recursive formula for computing $M: M(n)$ is obtained from $\mu(m)$ and $M(m)$ with $m<n$.

Theorem 9. Let $n$ and $r$ two integers satisfying $3 \leq r \leq n-1$. Then

$$
2 M(n)+3=\sum_{k=1}^{\left\lfloor\frac{n}{r+1}\right\rfloor} g(n, k) \mu(k)+\sum_{a=3}^{r} h(a)\left(M\left(\frac{n}{a}\right)-M\left(\frac{n}{a+1}\right)\right) .
$$

Proof. By (10) and (15),

$$
\begin{aligned}
2 M(n)+3 & =\sum_{k=1}^{\left\lfloor\frac{n}{3}\right\rfloor} g(n, k) \mu(k)=\sum_{k=1}^{\left\lfloor\frac{n}{r+1}\right\rfloor} g(n, k) \mu(k)+\sum_{a=3}^{r} \sum_{k=\left\lfloor\frac{n}{a+1}\right\rfloor+1}^{\left\lfloor\frac{n}{a}\right\rfloor} g(n, k) \mu(k) \\
& =\sum_{k=1}^{\left\lfloor\frac{n}{r+1}\right\rfloor} g(n, k) \mu(k)+\sum_{a=3}^{r} h(a) \sum_{k=\left\lfloor\frac{n}{a+1}\right\rfloor+1}^{\left\lfloor\frac{n}{a}\right\rfloor} \mu(k)
\end{aligned}
$$




$$
=\sum_{k=1}^{\left\lfloor\frac{n}{r+1}\right\rfloor} g(n, k) \mu(k)+\sum_{a=3}^{r} h(a)\left(M\left(\frac{n}{a}\right)-M\left(\frac{n}{a+1}\right)\right) .
$$

Prior to continue, let us note which would be the two limit cases: of course, $r=2$ is Theorem 6; and, when $r=n$, the sum indexed by $k$ in (9) disappears. Another particular case appears by taking $r=\lfloor\sqrt{n}\rfloor$; thus (17) becomes

$$
\begin{aligned}
M(n)=\frac{1}{2}\left(-3+\sum_{k=1}^{\left\lfloor\frac{n}{\lfloor\sqrt{n}\rfloor+1}\right\rfloor}\right. & g(n, k) \mu(k)+M\left(\frac{n}{3}\right) \\
& \left.+\sum_{a=4}^{\lfloor\sqrt{n}\rfloor}(h(a)-h(a-1)) M\left(\frac{n}{a}\right)-h(\lfloor\sqrt{n}\rfloor) M\left(\frac{n}{\lfloor\sqrt{n}\rfloor+1}\right)\right),
\end{aligned}
$$

a formula that resembles (2) after isolating $M(n)$, but starting in $M\left(\frac{n}{3}\right)$ instead of $M\left(\frac{n}{2}\right)$.

On the other hand, by splitting again the summand on the right in (17), we can introduce a new parameter $s$ :

Theorem 10. Let $n, r$ and $s$ be three integers such that $s \geq 0$ and $6 s+9 \leq r \leq n-1$. Then

$$
\begin{aligned}
2 M(n)+3=\sum_{k=1}^{\left\lfloor\frac{n}{r+1}\right\rfloor} g(n, k) \mu(k) & \\
+\sum_{b=0}^{s}\left(M\left(\frac{n}{3+6 b}\right)-2 M\left(\frac{n}{5+6 b}\right)+\right. & \left.3 M\left(\frac{n}{6+6 b}\right)-2 M\left(\frac{n}{7+6 b}\right)\right) \\
& +\sum_{a=6 s+9}^{r} h(a)\left(M\left(\frac{n}{a}\right)-M\left(\frac{n}{a+1}\right)\right) .
\end{aligned}
$$

Proof. Let us expand the summand $\sum_{a=3}^{r}$ in (17). In this way,

$$
\begin{aligned}
2 M(n)+3= & \sum_{k=1}^{\left\lfloor\frac{n}{r+1}\right\rfloor} g(n, k) \mu(k)+h(3) M\left(\frac{n}{3}\right)-h(3) M\left(\frac{n}{4}\right) \\
& +h(4) M\left(\frac{n}{4}\right)-h(4) M\left(\frac{n}{5}\right)+h(5) M\left(\frac{n}{5}\right)-h(5) M\left(\frac{n}{6}\right) \\
& +h(6) M\left(\frac{n}{6}\right)-h(6) M\left(\frac{n}{7}\right)+h(7) M\left(\frac{n}{7}\right)-h(7) M\left(\frac{n}{8}\right) \\
& +h(8) M\left(\frac{n}{8}\right)-h(8) M\left(\frac{n}{9}\right)+\cdots \\
+h(6 s+8) M\left(\frac{n}{6 s+8}\right)-h(6 s+8) M\left(\frac{n}{6 s+9}\right) & +\sum_{a=6 s+9}^{r} h(a)\left(M\left(\frac{n}{a}\right)-M\left(\frac{n}{a+1}\right)\right) .
\end{aligned}
$$

By applying the values of $h$ according (16), the result follows.

Thus, in Theorem 10 we have presented a two-parametric family of recurrence relation for computing an isolated value of $M(n)$. They provide mixed ways to calculate $M(n)$ using, in part, previously computed (and stored) values of $M(m)$ for a certain values of $m$, and another part that must be explicitly computed. Eventually, a suitable election of parameters 
$r$ and $s$ (that may depend on $n$ ) will allow to get efficient methods of running this algorithm in a computer; at this point, it is clear that a careful implementation must be performed, taking into account the machine to be used. The idea to use this expansion is as follows: The terms in the first sum $\sum_{k=1}^{\left\lfloor\frac{n}{r+1}\right\rfloor}$ are directly evaluated. The second sum $\sum_{b=0}^{s}$ is computed by using previously computed and stored values of $M$. And the third sum

$$
\sum_{a=6 s+9}^{r} h(a)\left(M\left(\frac{n}{a}\right)-M\left(\frac{n}{a+1}\right)\right)=\sum_{a=6 s+9}^{r} h(a) \sum_{k=\left\lfloor\frac{n}{a+1}\right\rfloor+1}^{\left\lfloor\frac{n}{a}\right\rfloor} \mu(k)
$$

can be computed by using both methods, according the size of $n, r$ and $s$.

Finally, let us note which are the limit cases of the identity established by Theorem 10: $s=-1$ (Theorem 9); $s=-1$ and $r=2$ (Theorem 6$) ; r=n$ ( $\sum_{b}$ disappears); and $r=6 s+8$ ( $\sum_{k}$ disappears).

\section{The function $H(n, m)$. Periodicity}

In the previous sections (see Theorems 6, 9 and 10), we often obtain expressions with the form $\sum_{k=1}^{m} g(n, k) \mu(k)$. Thus, in this section, we define a new function $H(n, m)$ (for non-negative integers $n$ and positive integers $m$ ) by taking

$$
H(n, m):=\sum_{k=1}^{m} g(n, k) \mu(k)
$$

and we are going to study some of its properties. Also, we will use the following notation:

$$
C_{m}:=6 \cdot \operatorname{lcm}\{1,2, \ldots, m\} .
$$

First, let us see that, when we fix $m$ in the second variable of $H$, the function is periodic with period $C_{m}$.

Proposition 11. For every non-negative integer $t$, we have

$$
H\left(n+t C_{m}, m\right)=H(n, m) .
$$

Proof. By being $g(n+6 k t, k)=g(n, k)$ for $k=1,2, \ldots, m$, we have $g\left(n+C_{m} t, k\right)=g(n, k)$. Thus, the result follows.

The following result gives the value of $H(n, m)$ as a function of $M(m)$.

Proposition 12. For every non-negative integer $t$, we have

$$
\begin{aligned}
& H\left(0+t C_{m}, m\right)=2 M(m), \\
& H\left(1+t C_{m}, m\right)=2 M(m)-2, \\
& H\left(2+t C_{m}, m\right)=2 M(m), \\
& H\left(n+t C_{m}, m\right)=2 M(m)+3, \quad \text { if } 2<n \leq m .
\end{aligned}
$$

Proof. By Proposition 11, without loss of generality, we can suposse $t=0$. Then, it is enough for computing $H(n, m)$ for $0 \leq n \leq m$. First, let us analyze the cases $n=0,1,2,3$. By applying (12), we have

$$
\begin{aligned}
& H(0, m)=\sum_{k=1}^{m} g(0, k) \mu(k)=2 \sum_{k=1}^{m} \mu(k)=2 M(m), \\
& H(1, m)=\sum_{k=1}^{m} g(1, k) \mu(k)=0 \cdot \mu(1)+2 \sum_{k=2}^{m} \mu(k)=2 M(m)-2,
\end{aligned}
$$




$$
H(2, m)=\sum_{k=1}^{m} g(2, k) \mu(k)=0 \cdot \mu(1)+0 \cdot \mu(2)+2 \sum_{k=3}^{m} \mu(k)=2 M(m) .
$$

For $n$ verifying $2<n \leq m$, let us decompose

$$
H(n, m)=\sum_{k=1}^{\left\lfloor\frac{n}{3}\right\rfloor} g(n, k) \mu(k)+\sum_{k=\left\lfloor\frac{n}{3}\right\rfloor+1}^{n} g(n, k) \mu(k)+\sum_{k=n+1}^{m} g(n, k) \mu(k) .
$$

Now, in the first sum, let us apply (10); in the second sum, let us use that, for $\left\lfloor\frac{n}{3}\right\rfloor<k \leq n$ (i.e., $k \leq n<3 k$ ) we have $g(n, k)=0$ (see (12)); and, finally, for the third sum, let us note that $g(n, k)=2$ for $0 \leq n<n+1 \leq k$. In this way, (18) becomes

$$
H(n, m)=(2 M(n)+3)+0+2(M(m)-M(n))=2 M(m)+3 .
$$

\section{REFERENCES}

[1] Apostol TM. Introduction to Analytic Number Theory. New York: Springer-Verlag; 1976.

[2] Mertens F. Über eine zahlentheoretische Funktion. Sitzungsberichte Akad. Wiss. Wien IIa. 1897; 106: 761-830.

[3] Hermite C. Correspondence d'Hermite et de Stieltjes: publiée par le soins de B. Baillaud et H. Bourget. Vol. 1. Paris: Gauthier-Villars; 1905.

[4] Littlewood JE, Quelques conséquences de l'hypothèse que la fonction $\zeta(s)$ n'a pas de zéros dans le demi-plan $\operatorname{Re}(s)>\frac{1}{2}$. C. R. Acad. Sci. Paris. 1912; 154: 263-266.

[5] Titchmarsh EC. The Theory of the Riemann Zeta-Function. Oxford: At the Clarendon Press; 1967.

[6] Odlyzko AM, te Riele H. Disproof of the Mertens conjecture. J. Reine Angew. Math. 1985; 357: 138-160.

[7] Pintz J. An effective disproof of the Mertens conjecture. In: Journées Arithmétiques (Besançon, France, 1985). Astérisque. 1987; 147-148: 325-333, 346.

[8] Kotnik T, te Riele H. The Mertens conjecture revisited. In: Algorithmic number theory. Lecture Notes in Comput. Sci. 4076. Berlin: Springer; 2006. p. 156-167.

[9] el Marraki M. Fonction sommatoire de la fonction de Möbius, 3. Majorations asymptotiques effectives fortes. J. Théor. Nombres Bordeaux. 1995; 7: 407-433.

[10] Kotnik T, van de Lune J. On the order of the Mertens function. Experiment. Math. 2004; 13: 473-481.

[11] Landau E. Handbuch der Lehre von der Verteilung der Primzahlen. Leipzig \& Berlin: Teubner; 1909.

[12] Deléglise M, Rivat J. Computing the summation of the Möbius function. Experiment. Math. 1996; 5: 291-295.

[13] Neubauer G. Eine empirische Untersuchung zur Mertensschen Funktion. Numer. Math. 1963; 5: 1-13.

[14] Dress F. Fonction sommatiore de la fonction de Möbius. I. Majorations expérimentales. Experiment. Math. 1993; 2: 89-98.

[15] te Riele H. On the history of the function $M(x) / \sqrt{x}$ since Stieltjes. In: Thomas Jan Stieltjes: Euvres Complètes/Collected Papers. Vol. 1. Berlin: Springer-Verlag; 1993. p. 69-79.

Instituto Sagasta, Glorieta del Doctor Zubía s/n, 26003 Logroño, Spain

E-mail address: mbenit8@palmera.pntic.mec.es

Departamento de Matemáticas y Computación, Universidad de la Rioja, Edificio J. L. Vives, Calle Luis de Ulloa s/n, 26004 Logroño, Spain

E-mail address: jvarona@dmc.unirioja.es

$U R L:$ http://www.unirioja.es/dptos/dmc/jvarona/welcome.html

Received: January 242008

Revised: April 18, 2008

Accepted: September 6, 2008

\section{(c) Benito and Varona; Licensee Bentham Open.}

This is an open access article licensed under the terms of the Creative Commons Attribution NonCommercial License (http://creativecommons.org/licenses/by-nc/3.0/) which permits unrestricted, non-commercial use, distribution and reproduction in any medium, provided the work is properly cited. 